\title{
Organogel-nanoemulsion containing nisin and D-limonene and its antimicrobial activity
}

\author{
Weiya Bei, Yan Zhou, Xuya Xing, Mohamed Reda Zahi, Yuan Li, Qipeng Yuan and \\ Hao Liang*
}

State Key Laboratory of Chemical Resource Engineering, Beijing University of Chemical Technology, Beijing, China

OPEN ACCESS

Edited by:

Sahdeo Prasad,

The University of Texas MD Anderson

Cancer Center, USA

Reviewed by:

Amit Kumar Tyagi,

The University of Texas MD Anderson

Cancer Center, USA

Shivani Agarwal,

Northwestern University, USA

Parag A. Parekh,

The University of Texas MD Anderson

Cancer Center, USA

*Correspondence:

Hao Liang,

State Key Laboratory of Chemical

Resource Engineering, Beijing

University of Chemical Technology,

15 Beisanhuan East Road, Chaoyang

District, Beijing 100029, China starslh@163.com

Specialty section:

This article was submitted to

Food Microbiology,

a section of the journal

Frontiers in Microbiology

Received: 11 June 2015 Accepted: 07 September 2015

Published: 22 September 2015

Citation:

Bei W, Zhou Y, Xing X, Zahi MR, Li Y,

Yuan Q and Liang H (2015)

Organogel-nanoemulsion containing nisin and D-limonene and its

antimicrobial activity.

Front. Microbiol. 6:1010

doi: 10.3389/fmicb.2015.01010
The aim of this study was to investigate a novel delivery system containing D-limonene and nisin by food organogel-nanoemulsion and study its effect on the antimicrobial activity. Organogel-nanoemulsion containing with D-limonene and nisin or without nisin was prepared by a homogenization method. Factors that may affect the droplet size and stability of organogel-nanoemulsion such as pressure and surfactant to oil ratio (SOR) were studied. The average droplet size decreased with pressure, and the organogelnanoemulsion could achieve good stability at low SOR. Positive effects and outstanding antimicrobial activities of organogel-nanoemulsion containing with D-limonene and nisin were confirmed by minimal inhibitory concentrations comparison, growth curves of bacteria, scanning electron microscopy and determination of cell constituents' release. Furthermore, the organogel-nanoemulsion applied as food preservative in milk also shown excellent antimicrobial performance. Overall, the research described in the current article show that organogel-nanoemulsion containing with D-limonene and nisin may be an effective antimicrobial system for the production and preservation of food.

Keywords: D-limonene, nisin, organogel-nanoemulsion, antimicrobial activity, food preservation

\section{Introduction}

It is well known that microbial contamination has got wide attention, due to it may bring potential adverse effects to consumers (Oliveira et al., 2011). Under the impacts of globalization and quick distribution systems, these can have good impacts with profound and even fatal result (Ellis et al., 2012). Some natural antimicrobial substances were used jointly with new technologies to resolve the issues of pathogenic bacteria, which could improve food safety and product quality (Gálvez et al., 2010). Antimicrobial delivery systems were proposed as potential solutions to improve effectiveness of antimicrobials in food matrices by safeguarding antimicrobials from contacting food matrix components and releasing them incessantly (Xiao et al., 2011). D-limonene (4-isopropenyl-1-methylcyclohexene) is an ingredient of many citrus fruits and it is widely used in many fields, including cosmetics, foods, and other products. A number of researchers have proved that D-limonene had bactericide, antioxidant, chemo-preventative, and therapeutic activities (Gálvez et al., 2010). Its outstanding antimicrobial activities have already been proven against different species of food-related microorganisms, such as Staphylococcus aureus, Listeria monocytogenes, Salmonella enterica, Saccharomyces bayanus (Abi-Ayada et al., 2011). 
However, due to high hydrophobic and easy to oxidative degradation natures of $\mathrm{D}$-limonene, it is difficult in achieving an even dispersion in water and directly results in its loss of activity. It will require more D-limonene to get identical antimicrobial efficiencies in foods, especially in the areas such as the interface of two different phases (Zhang et al., 2014). Therefore, it is very important and necessary protect $\mathrm{D}$-limonene from chemical degradation and improve its water-solubility.

A number of approaches have been explored to improve these shortcomings and limitations of hydrophobic, oxidationprone biologically active compounds. Many research groups have focused on combinations of essential oils (EOs) with synergistic activity (Gutierrez et al., 2009), and the combinations of EOs with other natural antimicrobial(e.g., Nisin) could get effective antibacterial activity with a small amount, then the effect of negative sensory on foods can be mitigated significantly (Govaris et al., 2010). Nisin is a peptide, the product of some Lactococcus lactis subsp. It was introduced for the first time in the UK as a food preservative. It has been widely acknowledged and applied to daily use in many countries (Delves-Broughton et al., 1996). In addition, other research groups found that emulsification was also a good way to improve their solubilities and stabilities (Benjamin et al., 2012). In our previous work, it was found that D-limonene nanoemulsion with nisin prepared by catastrophic phase inversion (CPI) method have shown good stability and outstanding antimicrobial activity (Zhang et al., 2014). So on this occasion, we studied organogel-nanoemulsion containing with D-limonene and nisin on the basis of early research.

Organogels are semi-solid systems, including liquid oil trapped within a three-dimensional networked structure which is formed by the self-assembly of a low concentration of organogelator molecules in a variety of organic liquids (Hughes et al., 2009). Organogel technology has been applied in many industries, and its potentials continue to be developed. Hence, we know that organogels have bright future in the industrial applications, especially in food industry, for its ability to structure edible oils. To our best knowledge, there are no reports about the exploration of the impacts of nisin on the antibiosis effect of D-limonene organogel-nanoemulsion for achieving a better preservative effect, which is the purpose of this research. In addition, we also focus on developing a novel antimicrobial delivery system combining positive effect of these two antibacterial agents at the same time.

\section{Materials and Methods}

\section{Materials}

D-limonene was obtained from Florida Worldwide Citrus Products Group Inc. (Bradenton, FL, USA). Nisin $\left(10^{4} \mathrm{IU} / \mathrm{mL}\right)$ was purchased from Lanzhou Weiri Bio-Engineering Co., Ltd., (Lanzhou, China). Sucrose stearate; sorbitan monooleate (Tween80), glutaraldehyde, sodium chloride (purity $>99.5 \%$ ), kanamycin sulfate was supplied by the Sinopharm Chemical Reagent Co., Ltd. (Beijing, China). Deionized water was filtered prior to use. Peanut oil and fresh $2 \%$ reduced fat milk were purchased from supermarket.

\section{Preparation of Organogel-Nanoemulsions Containing D-Limonene and Nisin}

Nisin (180 mg) was dissolved in $3 \mathrm{~mL}$ deionized water as the aqueous phase. Oil phase (10 g) containing stearic acid $(7 \mathrm{~g})$, sucrose stearate $170(\mathrm{~S} 170 ; 5 \% \mathrm{w} / \mathrm{w})$, peanut oil (88 or $68 \% \mathrm{w} / \mathrm{w}$ ) and $\mathrm{D}$-limonene (0 or $20 \% \mathrm{w} / \mathrm{w})$ were heated and stirred $\left(80^{\circ} \mathrm{C}\right)$, until the solution was clear. Then the two phases were homogenized (HENC homogenizer, Shanghai, China), and then frozen and lyophilized (FD-1C-50, Beijing, China), to produce an organogel phase. Next, the prepared organogel phase $(10 \mathrm{~g})$ was placed in an $80^{\circ} \mathrm{C}$ water bath again to form a transparent oil phase, and dispersed into another water phase $(50 \mathrm{~mL})$ containing Tween 80 with stirring, followed by high pressure homogenization to form nanoemulsion.

\section{Measurement of Droplet Size Diameters}

Dynamic light scattering was used to measure the mean particle diameters of samples at $25^{\circ} \mathrm{C}$ (Zetasizer Nano-ZS90, Malvern Instruments, Malvern, UK).

\section{Antimicrobial Activity Microbial Strains and Growth Conditions}

Three food-related microorganisms included the Gram-positive bacteria S. aureus ATCC6538, and Bacillus subtilis ATCC6633, the Gram-negative bacteria Escherichia coli ATCC8739. All strains were supplied by China General Microbiological Culture Collection Center (Beijing, China), maintained at $4^{\circ} \mathrm{C}$ and incubated at $37^{\circ} \mathrm{C}$ (Zhang et al., 2014).

\section{Synergism Testing: Checkerboard Method}

The interactive inhibition of antimicrobial compounds in vitro was evaluated by broth dilution checkerboard method (Hemaiswarya et al., 2008; Zhang et al., 2014). D-limonene was diluted twofold in vertical orientation, while nisin was diluted twofold in horizontal orientation. Their respective concentrations were prepared corresponding to $1 / 2,1 / 4$, and $1 / 8$ of the minimal inhibitory concentration (MIC) values, respectively. Next, $400 \mu \mathrm{L}$ suspension containing $\left(1 \times 10^{8} \mathrm{CFU} / \mathrm{mL}\right)$ of the indicator microorganism was added to each tube and incubated overnight at $37^{\circ} \mathrm{C}$.

We calculated the fractional inhibitory concentration indices $(\mathrm{FICI})$ as follow: FICI $=$ FICA + FICB, where FICA $=(\mathrm{MICA}$ of the combination/MICA alone) and FICB $=$ (MICB of the combination/MICB alone). The results were divided into the following categories: synergy (FICI $\leq 0.5$ ), addition $(0.5 \leq \mathrm{FICI} \leq 1)$, indifference $(1 \leq \mathrm{FICI} \leq 4)$ or antagonism (FICI > 4) (Zhang et al., 2014).

\section{Determination of Minimal Inhibitory Concentration}

The double broth dilution method was adopted to confirm the MIC values with some modifications as described by previous researchers (Weerakkody et al., 2010). After adding appropriate antimicrobial agents to the first tube containing $4 \mathrm{~mL}$ of broth, serial twofold dilutions were made. A $400 \mu \mathrm{L}$ suspension $\left(1 \times 10^{8} \mathrm{CFU} / \mathrm{mL}\right)$ of designated microorganism was added to each tube. 
The tube contained only broth and microorganism as a negative control. Meanwhile, the tube contained broth, $50 \mu \mathrm{g} / \mathrm{mL}$ of kanamycin sulfate and microorganism as a positive control (Al-Reza et al., 2009; Lv et al., 2011).

\section{Growth Curves of Bacterium Treated with Organogel-Nanoemlusion}

Logarithmic phase cells were utilized in the tests, following by a dilution to prepare bacterium suspensions of $10^{5}-10^{6} \mathrm{CFU} / \mathrm{mL}$. The suspensions were dealt with different concentrations of organogel-nanoemulsions of D-limonene and nisin (control, MIC, $2 \times$ MIC and $4 \times$ MIC), then incubated in same suitable conditions. Cells were harvested by centrifugation $(5000 \mathrm{rpm}$ for $10 \mathrm{~min}$ ) every $2 \mathrm{~h}$. After three times of washing and collection, cells were re-suspended in phosphate buffer solution (PBS, $0.1 \mathrm{M}, \mathrm{pH}$ 7.0). The quantity of cells was measured by UVVis (SHIMADZU UV2450) (Govaris et al., 2010; Zhang et al., 2014).

\section{Scanning Electron Microscopy (SEM) Analysis}

Scanning electron microscopy (SEM) studies were adopted as reported by Moosavy et al. (2008) and Zhang et al. (2014) with some modifications. Three tested microorganisms (about $1 \times 10^{8} \mathrm{CFU} / \mathrm{mL}$ ), which in logarithmic growth phase, were treated with each MIC of organogel-nanoemlusion containing $\mathrm{D}$-limonene and nisin. Then samples were incubated at room temperature for $3 \mathrm{~h}$, and harvested by centrifugation and washed twice with PBS (0.1 M, pH 7.0). And then resuspended in PBS including $2.5 \%$ glutaraldehyde and kept for $2 \mathrm{~h}$ at $-4^{\circ} \mathrm{C}$. Followed by further dehydrated at different ethanol concentrations. At last, the thalli were fixed and sputter-coated with gold, then observed by SEM (Zeiss Supra ${ }^{7 M}$ 55, Stuttgart, Germany) (Lv et al., 2011; Zhang et al., 2014).

\section{Determination of the Release of Cell Constituents}

The release of cell constituents was inspected through the method previously described (Rhayour et al., 2003; Lv et al., 2011; Zhang et al., 2014). Individual cultures were grown for $24 \mathrm{~h}$ in their respective medium, after which $100 \mathrm{~mL}$ was collected and centrifuged, washed three times with PBS, and finally resuspended in $\mathrm{PBS}(0.1 \mathrm{M}, \mathrm{pH}$ 7.0). The washed cell suspensions were incubated at $37^{\circ} \mathrm{C}$ in an environmental incubator shaker for $1 \mathrm{~h}$ after treated by different concentration of organogelnanoemulsion (control, MIC and $2 \times \mathrm{MIC}$ ). Then, $2 \mathrm{~mL}$ of samples were collected and centrifuged, and the centrifuged cell supernatant was used to measure UV absorption at $260 \mathrm{~nm}$.

\section{Determination of Antimicrobial Activity of Oraganogel-Nanoemulsions with Nisin and D-Limonene in Milk}

In the test, fresh $2 \%$ reduced fat milk was chosen as the target food to assess antimicrobial performance of organogel-nanoemusions with $\mathrm{D}$-limonene and nisin. The milk samples were incubated at room temperature for 5 days in the presence of different conditions of organogel-nanoemulsions (control and $2 \times \mathrm{MIC}$ ). After incubating the mixtures at room temperature for $120 \mathrm{~h}$, the pour plate method was used to enumerate the viable bacteria (Ma et al., 2013).

\section{Statistical Analysis}

All experiments were performed in triplicate. The data were recorded as mean \pm standard deviation for the measurements. Differences between means were considered significant at $p<0.05$. The SPSS programmer (SPSS, version 12.0 for Windows, SPSS Inc.) was used to analyze the results.

\section{Results}

\section{Preparation and Characterizations of Organogel-Nanoemulsions with D-Limonene and Nisin}

In order to achieve homogeneous and stable organogelnanoemulsions, and establish the most appropriate surfactant to oil ratio (SOR) and pressure, which contribute to the formulation, size distribution and, more importantly, stability of organogelnanoemulsions, the preparation process was optimized at first, a series of emulsion processes were prepared under different pressures and SOR values. All the nanoemulsion samples contained D-limonene and nisin of $6 \%, 15 \%(w / w)$. For pressure optimization, a wide range of preparation pressures $(30,50$, 80 , and $100 \mathrm{MPa}$ ) from high pressure homogenizer were utilized to explore and compare the particle size distributions. As the results shown in Figure 1, both Volume (\%) value (Figure 1A) and Intensity (\%) value (Figure 1B) of particle size distributions of all samples displayed bimodal results, in which the vast majority of the droplet population had a dropletsize distribution around 100 or $10 \mathrm{~nm}$, only a small population around $10 \mu \mathrm{m}$. The highest homogenizing pressure of $100 \mathrm{MPa}$ prepared the minimum droplet size of $10 \mathrm{~nm}$ by Volume (\%) value (Figure 1A), which could provide large specific surface area and stable capacity for the following antimicrobial experiments of organogel-nanoemulsions.

During the SOR of optimization process, we chose food-grade Tween 80 as the surfactant, prepared a series of nanoemulsions with different SORs (from 1:8 to $1: 3$ ) and same homogenizing pressure of $100 \mathrm{MPa}$. The results in Figure 2 show that all the samples exhibit similar drop size distributions with a main population around in $100 \mathrm{~nm}$ and a mall population in $10 \mathrm{~nm}$. The results indicate that organogel-nanoemulsions prepared with very low concentration of surfactant (SOR 1:8) could achieve narrow and uniform particle size distribution, contributing to a low surfactant additions in food.

In our previous study, D-limonene organogel-based nanoemulsion exhibited a good stability: the particle size increased $10 \mathrm{~nm}$ at $28^{\circ} \mathrm{C}$ and $6 \mathrm{~nm}$ at $4^{\circ} \mathrm{C}$ during a storage period of 2 weeks (Zahi et al., 2014). In this study, we got similar and even better results. The results in Figure 3 show that the storage stability of organogel-nanoemulsions prepared with different pressures (Figure 3A) and SORs (Figure 3B). There was only a very slight increase in the average size for all samples stored at $28^{\circ} \mathrm{C}$, which confirmed that organogelnanoemulsion containing $\mathrm{D}$-limonene and nisin has an excellent 

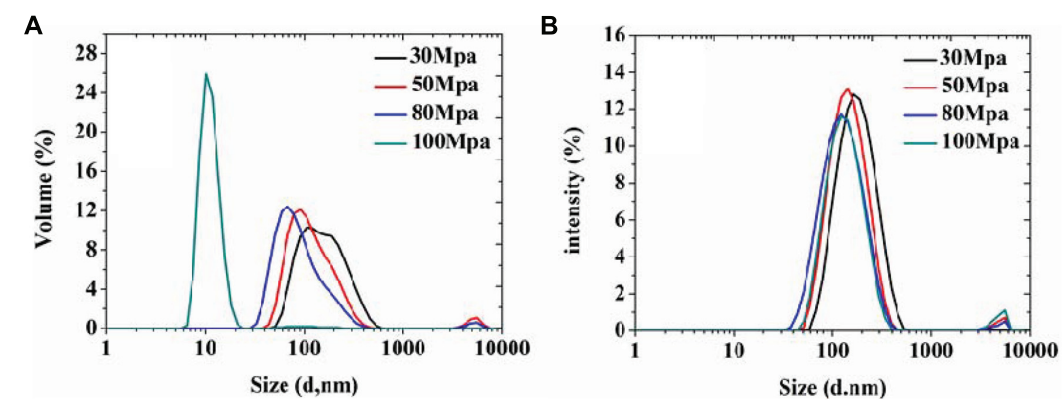

FIGURE 1 | The particle size distributions of organogel-nanoemulsion prepared under different homogenizing pressures were analyzed by DLS [size distributions by volume (A) and intensity (B)].
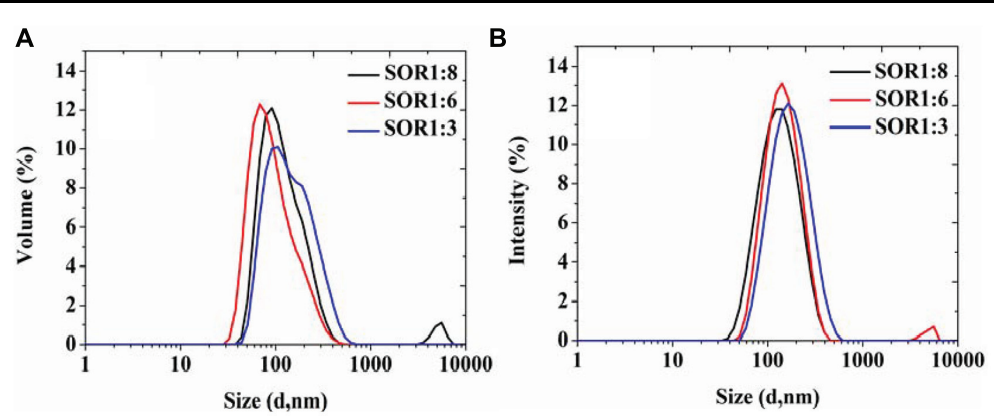

FIGURE 2 | The particle size distributions of organogel-nanoemulsion prepared under different SORs were analyzed by DLS [size distributions by volume (A) and intensity (B)].
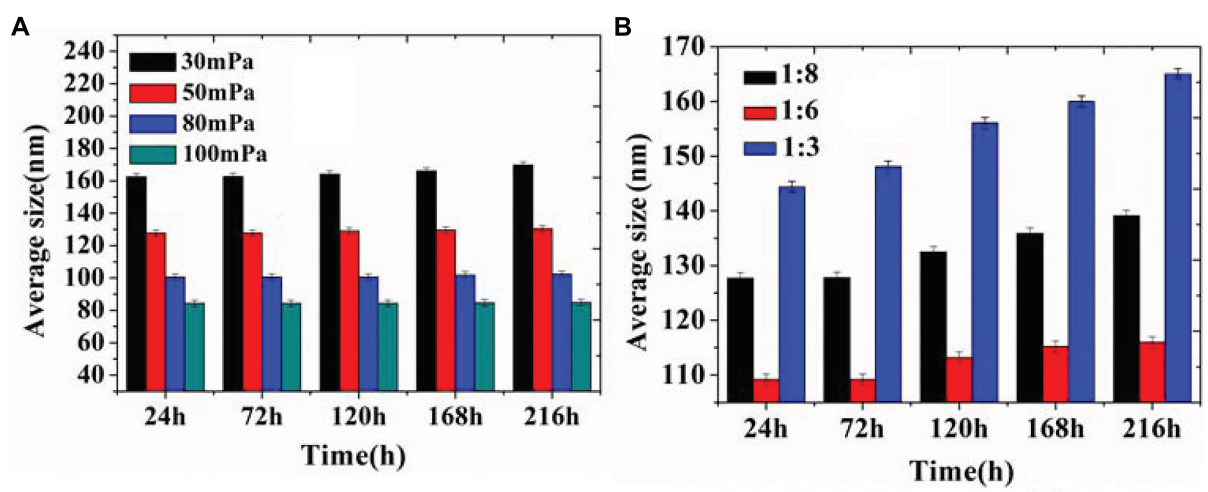

FIGURE 3 | Storage stability of organogel-nanoemulsion prepared under different pressures (A) and SORs (B) at $28^{\circ} \mathrm{C}$.

stability once more. All the samples were stored at $28^{\circ} \mathrm{C}$ for no fewer than 3 months showed no stratification or phase separation.

\section{In Vitro Synergy between Nisin and D-Limonene}

To enhance the antagonistic activity and as such reduce the quantity of preservatives added to food, nisin has been found to act in synergy with various antimicrobial agents including cheaters, oregano EO, reuterin, and phage endolysin (Govaris et al., 2010). In this work, three food-related microorganisms
(S. aureus; B. subtilis, and E. coli) were chosen as the target bacterium. When nisin and D-limonene were combined by the checkerboard method, synergistic and additive effects were observed (Table 1). With reference to the FICI scale, nisin and D-limonene combination displayed a synergism with regards to S. aureus and B. subtilis (FICI $=0.375)$, and exhibited a useful additive effect with the FICI of 1.0 for the E. coli. No indifference and antagonistic effects were observed (Zhang et al., 2014). The synergistic and additive effects between D-limonene and nisin could provide a strong support for the next tests of antibacterial of organogel-nanoemulsion. 
TABLE 1 | Fractional inhibitory concentration indices (FICI) of combinations of D-limonene and nisin against the tested microorganisms ${ }^{a, b}$.

\begin{tabular}{|c|c|c|c|c|c|c|}
\hline \multirow[t]{2}{*}{ Combination } & \multicolumn{2}{|c|}{ Staphylococcus aureus } & \multicolumn{2}{|c|}{ Bacillus subtilis } & \multicolumn{2}{|c|}{ Escherichia coli } \\
\hline & FIC & FICI & FIC & $\mathbf{F I C l}$ & FIC & $\mathbf{F I C l}$ \\
\hline \multirow[t]{3}{*}{ D-limonene-nisin } & 0.25 & $0.37(S)$ & 0.25 & 0.37 (S) & 1.0 & $1.0(\mathrm{~A})$ \\
\hline & 0.125 & & 0.125 & & 0 & \\
\hline & 5 & & 5 & & & \\
\hline
\end{tabular}

a Results are means of three different experiments.

b The results were interpreted as synergy (S, FICl < 0.5), addition (A, $0.5 \leq \mathrm{FICl} \leq 1)$, indifference $(\mathrm{l}, 1<\mathrm{FICl} \leq 4)$ or antagonism (AN, $\mathrm{FICl} \geq 4)$.

\section{Antimicrobial Activity of Organogel-Nanoemulsions}

As the results of MIC values shown in the Table 2, the MICs of organogel-nanoemulsions containing both $\mathrm{D}$-limonene and nisin (D-limonene 15\% w/w and nisin 6\% w/w) decreased obviously comparing to other two kinds of organogel-nanoemulsions containing only D-limonene $(15 \% \mathrm{w} / \mathrm{w})$ or only nisin $(6 \% \mathrm{w} / \mathrm{w})$ against all the three target microorganisms. More importantly to the Gram-negative E. coli, organogel-nanoemulsions with D-limonene and nisin displayed a strong enhancement (MIC $42.15 \mu \mathrm{g} / \mathrm{mL}$ ) than the organogel-nanoemulsions with only nisin (MIC > $5000 \mu \mathrm{g} / \mathrm{mL}$ ). As we know nisin had no antimicrobial effect on any of Gram-negative bacteria such as E.coli. The result maybe directly attributed to the additive effect of D-limonene with nisin in the system of organogelnanoemulsions. Furthermore, the addition of two antibacterial agents also decreased significantly according to the MICs of D-limonene and nisin, respectively, shown in the Table 2, especially for the $S$. aureus (MICs of D-limonene and nisin decreased from 4.75 to $1.1 \mu \mathrm{g} / \mathrm{mL}, 0.45$ to $0.33 \mu \mathrm{g} / \mathrm{mL}$ ) and B. subtilis (MICs of D-limonene and nisin decreased from 4.38 to $2.2 \mu \mathrm{g} / \mathrm{mL}, 0.9$ to $0.66 \mu \mathrm{g} / \mathrm{mL}$ ).

\section{Growth Curves of Bacteria and Antimicrobial Activity in Milk}

In order to further verify the antibacterial effects of organogelnanoemulsions growth curve of three target microorganisms were detected under different conditions in this study. The strains were treated with 0 , MIC, $2 \times$ MIC and $4 \times$ MIC of organogel-nanoemulsions containing D-limonene $(15 \%$ $\mathrm{w} / \mathrm{w})$ and nisin $(6 \% \mathrm{w} / \mathrm{w})$. After incubation for $168 \mathrm{~h}$, the growth cures were summarized in Figure 4. As shown in Figure 4, organogel-nanoemulsions containing D-limonene and nisin displayed significant inhibitory effects against all the target microorganisms, especially for E.coli which shown the best results (Figure 4A). Comparing with blank samples of uncontrolled growth, organogel-nanoemulsions containing D-limonene and nisin effectively inhibited the division and reproduction of logarithmic phase of bacterium. The number of living bacterium decreased obviously under the treatments of organogel-nanoemulsions. Furthermore, the growth corves under treatment couldn't reach the normal growth peak any more but quickly enter into decline phase, especially for the $4 \times$ MIC which shown basically no obvious growth in number of bacterium (Figure 4).

In order to assess preservative effect of the organogelnanoemulsions with D-limonene and nisin in food, determinations of colony-forming units of bacterium in $2 \%$ reduced fat milk have been conducted under different concentrations of organogel-nanoemulsions with D-limonene and nisin (control and $2 \times \mathrm{MIC}$ ). After incubating for $120 \mathrm{~h}$ at room temperature for $48 \mathrm{~h}$, colony-forming units of blank and $2 \times$ MIC treated milk samples have been calculated and presented in Figure 5. As shown in the Figure 5, blank milk sample displayed a rapid growth from $72 \mathrm{~h}$ and finally reached colony-forming units as many as $7.50 \mathrm{E}+011 \mathrm{CFU} / \mathrm{mL}$ at $120 \mathrm{~h}$. However, due to the inhibition influence of $2 \times$ MIC organogel-nanoemulsions, test milk sample shown not only slower growth rate but also limited increasing amount of units (with a final colony-forming units at $1.50 \mathrm{E}+011 \mathrm{CFU} / \mathrm{mL}$ around).

\section{Mechanisms of Action of Organogel-Nanoemulsion with Nisin and D-Limonene Against Cell Membranes}

In order to visualize the effects of D-limonene organogelnanoemulsion with and without nisin against the cell membranes of the microbial cultures, we employed SEM of

TABLE 2 | Minimal inhibitory concentrations (MIC) values of three kinds of organogel-nanoemulsions against three target microorganisms.

\begin{tabular}{llll}
\cline { 2 - 4 } & $\begin{array}{l}\text { Organogel-nanoemulsion } \\
\text { (D-limonene 15\%) }\end{array}$ & $\begin{array}{l}\text { Organogel-nanoemulsion } \\
\text { (Nisin 6\%) }\end{array}$ & $\begin{array}{l}\text { Organogel-nanoemulsion } \\
\text { (D-limonene 15\% and Nisin 6\%) }\end{array}$ \\
\hline S. aureus & $23.75^{\mathrm{a}}(4.75)^{\mathrm{b}}$ & $7.5^{\mathrm{a}}(0.45)^{\mathrm{b}}$ & $5.47^{\mathrm{a}}(1.1,0.33)^{\mathrm{b}}$ \\
B. subtilis & $21.88(4.38)$ & $15(0.9)$ & $10.94(2.2,0.66)$ \\
E. coli & $43.75(8.75)$ & $>5000$ & $42.15(8.75,2.65)$
\end{tabular}

${ }^{a}$ The MICs of organogel-nanoemulsions against three target microorganisms.

${ }^{\mathrm{b}}$ The MICs of D-limonene or/and nisin in organogel-nanoemulsions against three target microorganisms. 

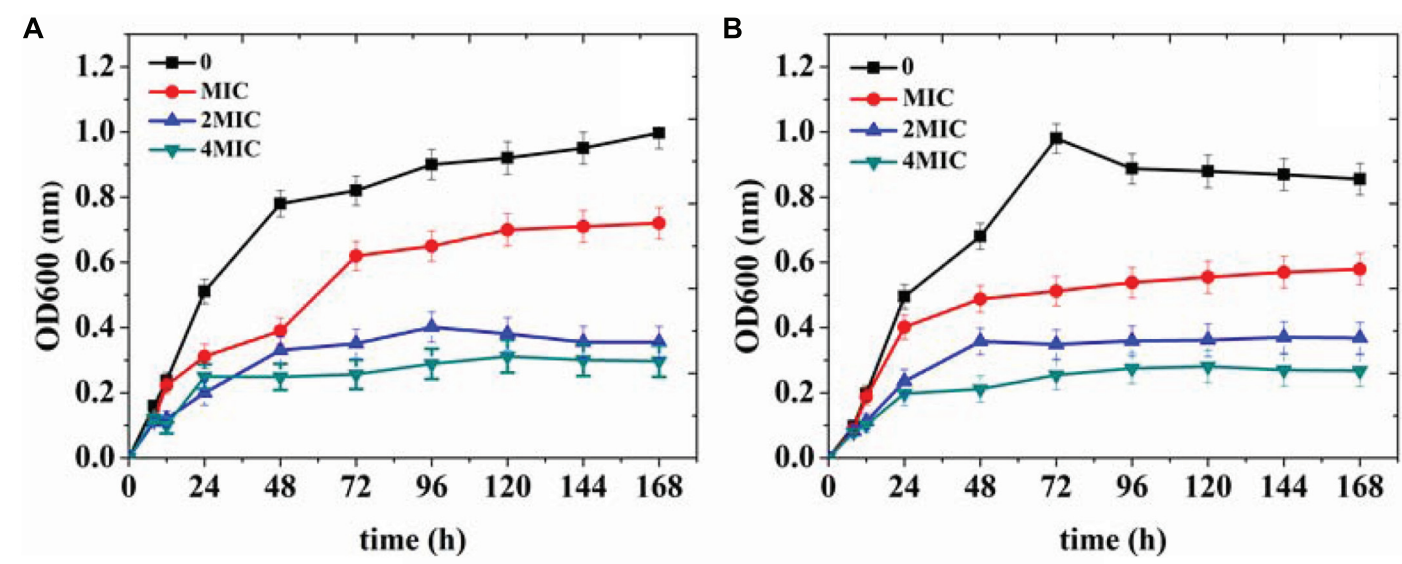

C

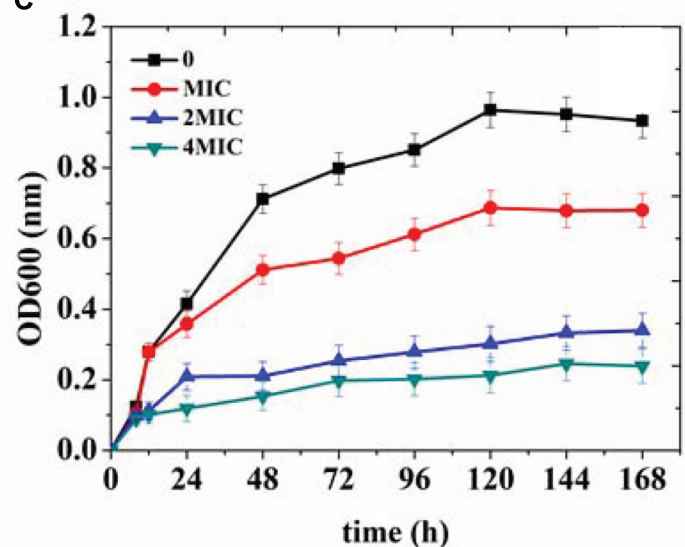

FIGURE 4 | Growth curves of Escherichia coli (A), Staphylococcus aureus (B), and Bacillus subtilis (C) treated with different concentrations [control, minimal inhibitory concentrations (MIC), $2 \times$ MIC, $4 \times$ MIC] of organogel-nanoemulsion containing D-limonene and nisin.

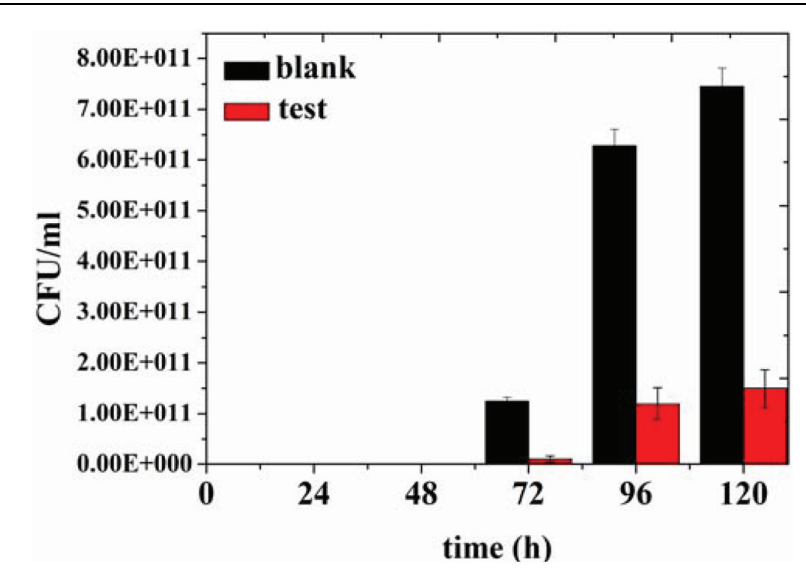

FIGURE 5 | Colony-forming units of bacteria in $2 \%$ reduced fat milk by different concentration of $\mathrm{D}$-limonene organogel-nanoemulsion with nisin (control, $2 \times$ MIC).

cells treated at the previously established MIC values, of Dlimonene organogel-nanoemulsion (D-limonene $20 \% \mathrm{w} / \mathrm{w}$ ), and $\mathrm{D}$-limonene organogel-nanoemulsion with nisin (D-limonene
20\%, nisin 6\% w/w). As shown in Figure 6, a different degree of deformation and distortion was observed following the addition of D-limonene organogel- nanoemulsion in to the three microorganisms (Figures 6B,E,H). However, all microorganisms exposed to the combined treatment of D-limonene organogelnanoemulsion with nisin suffered an almost serious collapse of the cell structure together with cell lysis (Figures 6C,F,I), which should be attributed to their good synergism against this Gram-negative bacteria, This can demonstrate its outstanding detrimental antimicrobial activity on the cellular integrity of all microorganisms tested. It is also noteworthy that the organogelnanoemulsion with the inclusion of nisin had no antimicrobial effect on E.coli (Figure 6J), which is in accordance with the MIC results.

Furthermore, we measured the release of cellular content from the damaged cells as evidence that the cell membranes were damaged, and the cell constituents are released. The target microorganisms were treated with 0 , MIC and $2 \times \mathrm{MIC}$ of $\mathrm{D}$-limonene organogel-nanoemulsion with nisin and incubated for $1 \mathrm{~h}$. Our results showed that the exposure to D-limonene organogel-nanoemulsion with nisin caused the release of cell constituents, which increased with the increasing concentration of D-limonene organogel-nanoemulsion with nisin as compared 

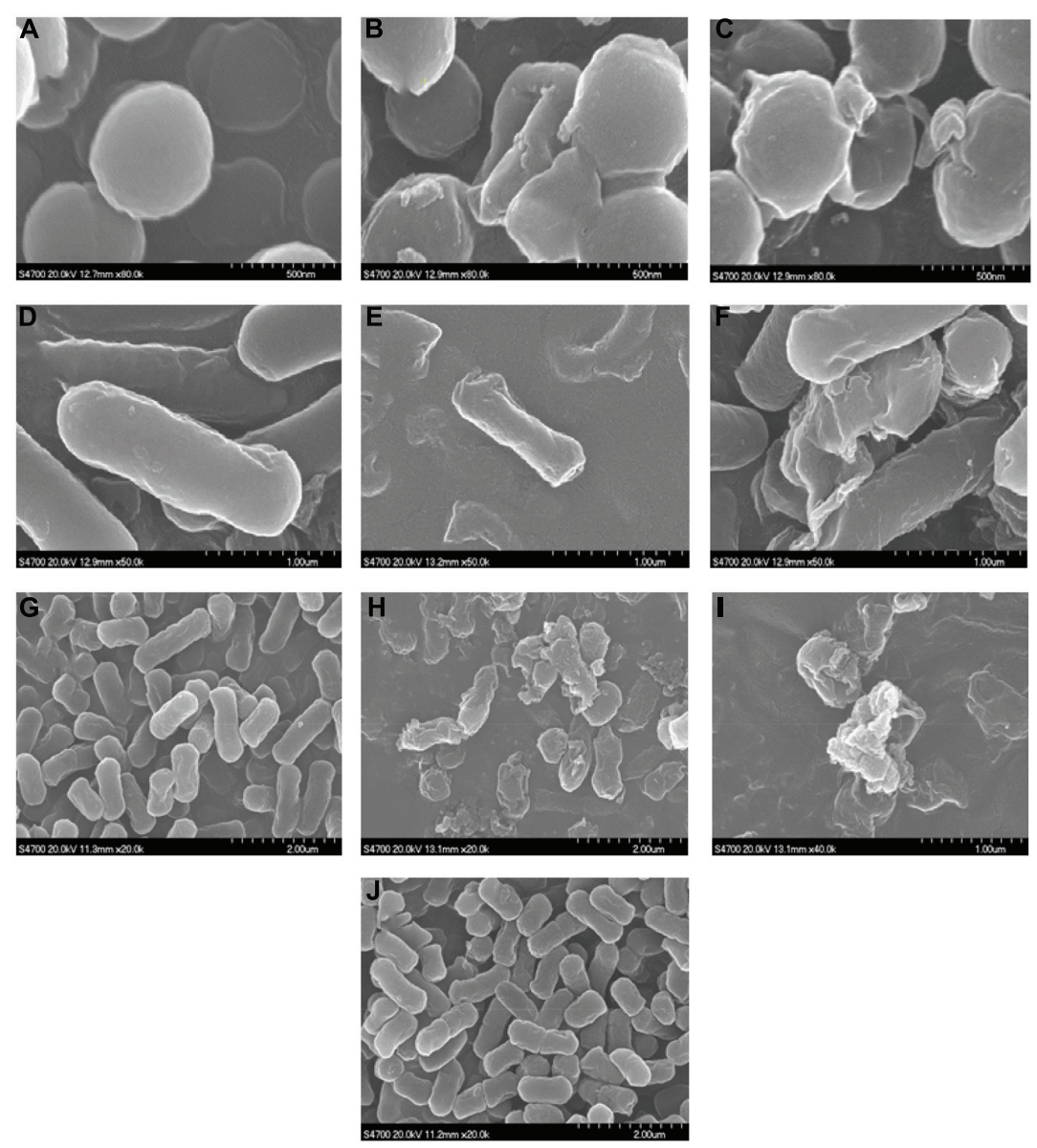

FIGURE 6 | Scanning electron micrographs of S. aureus (A-C), B. subtilis (D-F), and (G-J) cells: control (A,D,G), treated with D-limonene organogel -nanoemulsion (B,E,H), treated with D-limonene organogel-nanoemulsion with nisin (C,F,I), and E.coli treated with organogel-nanoemulsion with nisin (J), at MIC value for $3 \mathrm{~h}$ (magnification $\times 30,000$ or 50,000 ).

TABLE 3 | The effect of organogel-emulsification on cell constituents' release of the tested microorganisms.

\begin{tabular}{lll}
\hline Microorganism & $\begin{array}{l}\text { Nanoemulsions } \\
\text { concentration }\end{array}$ & $\begin{array}{l}\text { Cell constituents' release } \\
\left(\mathbf{O D}_{\mathbf{2 6 0 n m}}\right)^{\mathbf{a}}\end{array}$ \\
\hline B. subtilis & 0 & $0.033 \pm 0.004^{\mathrm{b}}$ \\
& MIC & $0.179 \pm 0.011$ \\
E. coli & MIC $\times 2$ & $0.451 \pm 0.008$ \\
& 0 & $0.013 \pm 0.003$ \\
S. aureus & MIC & $0.198 \pm 0.012$ \\
& MIC $\times 2$ & $0.314 \pm 0.022$ \\
& 0 & $0.021 \pm 0.004$ \\
& MIC & $0.129 \pm 0.009$ \\
& MIC $\times 2$ & $0.337 \pm 0.018$
\end{tabular}

a Results are presented as mean \pm standard deviation from the experiments in triplicate. $O D_{260 n m}$, optical density at $260 \mathrm{~nm}$.

bMeans within a same strain, which are not followed by a common letter, are significantly different $(P<0.05)$.

to the control group (Table 3). The most effective release of cellular constituents was observed following the $2 \times$ MIC treatment of B. subtilis. The magnitude of the release of cellular constituents (Table 3) appears to correspond well to the observed damaged by SEM (Figure 6).

\section{Discussion}

The use of D-limonene and nisin, as antimicrobials is appealing because these compounds are a "natural" alternative to traditional treatment methods. The effectiveness of D-limonene against various foodborne pathogens has been reported in numerous studies (Gálvez et al., 2010; Abi-Ayada et al., 2011). However, D-limonene is hydrophobic and therefore has low watersolubility. The organogel-nanoemulsion process utilized in this, allowed for the dispersion of D-limonene into aqueous phases in the form of small droplets. In a water-dispersible form, Dlimonene was able to act on any pathogens also present in the surrounding aqueous phase or at surfaces. And furthermore, D-limonene used in conjunction with nisin in this study.

We adopted high-pressure homogenization method to prepare organogel-nanoemulsion in this study, which is differ from CPI method that we have employed (Zhang et al., 2014). 
Furthermore, we incorporated D-limonene into the organogel prior to homogenization, which could immobilize the oil and improve the dispersibility of D-limonene in an easy and rapid way. Organogel-nanoemulsion was considered to combine the advantages of organogel and nanoemulsion, not only improved the solubility, but also enhanced the stability of hydrophobic compounds. Organogel-nanoemulsion can be used as a kind of excellent delivery system, especially in water-rich systems.

Droplet size is one of the most important indicators, in the assessment of emulsion performance, including the particle size and size distribution, which directly influence the stability of the emulsion and the sensory properties, function characteristics, shelf life of product (Floury et al., 2000; Benjamin et al., 2012). In this study, pressure and SOR are the main factors influencing the emulsion particle size and size distribution in the process of high pressure homogenizing. Our results demonstrated that pressure was higher, the smaller the particle size, the higher the degree of droplet refinement and distribution more uniform (Figure 1). Due to vast addition of surfactants in emulsion could significantly influence the quality of appearance and taste of food. So we have taken this constraint into consideration and made sure that in addition to gaining stable nanoemulsion, the SOR could be minimum at the same time. The results indicate that organogel-nanoemulsions prepared with very low concentration of surfactant (SOR 1:8) could achieve narrow and uniform particle size distribution (Figure 2). In addition, the prepared organogel-nanoemulsions showed outstanding storage stability (Figure 3). The small increase in the average size for all samples stored at $28^{\circ} \mathrm{C}$ is maybe due to the movement of the dispersed droplet pervaded the dispersing phase, then the chances of droplet collisions increased (Zahi et al., 2014).

Due to the results of synergistic or additive effect between D-limonene and nisin above, we prepared organogelnanoemulsions containing these two synergistic antibacterial agents to determinate if there are any improvements of antimicrobial performance. At the same time organogelnanoemulsions containing only D-limonene and only nisin were also prepared with same content and conditions in order to easily compare the antibacterial activity. To begin with, organogelnanoemulsions control sample (without nisin or D-limonene) had no antimicrobial effect on any of the microorganisms tested here. The results (Table 2) have proved that the combination of D-limonene and nisin has improved the antimicrobial proprieties significantly. Lower MICs of antimicrobial agents not only contributes to stronger antibacterial performance of the organogel-nanoemulsions, but also result in a smaller addition to the food products when applying as preservatives.

According to the aim of this study, we also pay much attention to safety problem in food. There is a need to survey the food safety performance in the agri-food chain without performing actual microbiological analysis. European food businesses have developed and validated seven indicators and corresponding assessment grids according to an extensive microbiological assessment scheme (MAS), which provided a foundation for the food safety performance diagnosis (Tajkarimi et al., 2010). The result provides positive evidence in favors of the argument that the combined application of D-limonene organogel-nanoemulsion with the inclusion of nisin imparts an excellent antimicrobial activity when applying in food.

Although the antimicrobial effects of different EOs have been shown by various microorganisms (Al-Reza et al., 2009; Govaris et al., 2010; Tajkarimi et al., 2010; Chikhoune et al., 2013), the mechanism has not been researched at length. Chemical analysis of series of EOs showed that the primary antimicrobial active components were phenols, terpenes, aldehydes, and ketones. There is a popular belief that EOs mainly acts against the cell cytoplasmic membrane. The principal mechanisms of action of D-limonene and nisin are against the cytoplasmic membranes of microorganisms, resulting in the loss of membrane integrity; the inhibition of respiratory enzymes; and dissipation of the proton-motive force (Sikkema et al., 1995; Rhayour et al., 2003; Sun, 2007). We extended our investigation to further uncover the mechanisms that underpin the action of D-limonene based organogel-nanoemlusion with nisin. The result provides positive evidence in favor of the argument that the combined application of $\mathrm{D}$-limonene organogel-nanoemulsion with the inclusion of nisin imparts an excellent antimicrobial activity.

The utilization of D-limonene organogel-nanoemulsion containing nisin prepared by high-pressure homogenization method displayed good physical stability and antimicrobial activity. In addition, growth curves of bacteria have shown that D-limonene organogel-nanoemulsion with nisin inhibits the reproduction of bacteria. SEM and the cells release determination revealed that $\mathrm{D}$-limonene organogel-nanoemulsion with nisin demolished the integrity of the cells' membrane, then causing the death of the microorganisms. This current study combined the advantages of organogel technology and the synergistic effects of multiple antimicrobial compounds in one system, not only widening the antimicrobial spectrum of nisin but also obviously reducing the amount of $\mathrm{D}$-limonene and nisin. The combined use of $\mathrm{D}$-limonene and nisin may provide a label friendly alternative to some of the currently used methods. The use of D-limonene organogel-nanoemulsion containing nisin as an antimicrobial treatment is relatively new and requires further studies. The interaction between D-limonene organogel-nanoemulsion with nisin and various food compounds and matrices has to be studied to better understand the physiochemical properties of this system. With greater understanding of the system as a whole, the use of antimicrobial organogel-nanoemulsion may find a broad range of applications within the production, processing, service and consumption of foods and beverages.

\section{Acknowledgment}

This study was supported by the National High Technology Research and Development Program of China (863 Program, GrantNos. 2012AA021403 and 2014AA021705), the Beijing Higher Education Young Elite Teacher Project (YETP0520), the Fundamental Research Funds for the Central Universities (YS1407) and China Scholarship Council. 


\section{References}

Abi-Ayada, F. Z., Abi-Ayada, M., Lazzounia, H. A., and Rebiahib, S. A. (2011) Antibacterial Activity of Essential oil extracted from leaves of Tetraclinis articulata (Vahl) Master from Algeria flora. J. Microbiol. Biotechnol. Res. 1, 1-6.

Al-Reza, S. M., Bajpai, V. K., and Kang, S. C. (2009). Antioxidant and antilisterial effect of seed essential oil and organic extracts from Zizyphus jujuba. Food Chem. Toxicol. 47, 2374-2380. doi: 10.1016/j.fct.2009.06.033

Benjamin, O., Silcock, P., Leus, M., and Everett, D. W. (2012). Multilayer emulsions as delivery systems for controlled release of volatile compounds using $\mathrm{pH}$ and salt triggers. Food Hydrocoll. 27, 109-118. doi: 10.1016/j.foodhyd.2011. 08.008

Chikhoune, A., Hazzit, M., Kerbouche, L., Baaliouamer, A., and Aissat, K. (2013). Tetraclinis articulata (Vahl) Masters essential oils: chemical composition and biological activities. J. Essent. Oil Res. 25, 300-307. doi: 10.1080/10412905.2013.774625

Delves-Broughton, J., Blackburn, P., Evans, R. J., and Hugenholtz, J. (1996). Applications of the bacteriocin, nisin. Antonie Van Leeuwenhoek 69, 193-202. doi: 10.1007/BF00399424

Ellis, D. I., Brewster, V. L., Dunn, W. B., Allwood, J. W., Golovanov, A. P., and Goodacre, R. (2012). Fingerprinting food: current technologies for the detection of food adulteration and contamination. Chem. Soc. Rev. 41, 5706-5727. doi: $10.1039 / \mathrm{C} 2 \mathrm{CS} 35138 \mathrm{~B}$

Floury, J., Desrumaux, A., and Lardières, J. (2000). Effect of high pressure homogenization on droplet size distributions and rheological properties of model oil-in-water emulsions. Innov. Food Sci. Emerg. Technol. 1, 127-134. doi: 10.1016/S1466-8564(00)00012-6

Gálvez, A., Abriouel, H., Benomar, N., and Lucas, R. (2010). Microbial antagonists to food-borne pathogens and biocontrol. Curr. Opin. Biotechnol. 21, 142-148. doi: 10.1016/j.copbio.2010.01.005

Govaris, A., Solomakos, N., Pexara, A., and Chatzopoulou, P. S. (2010). The antimicrobial effect of oregano essential oil, nisin and their combination against Salmonella Enteritidis in minced sheep meat during refrigerated storage. Int. J. Food Microbiol. 137, 175-180. doi: 10.1016/j.ijfoodmicro.2009.12.017

Gutierrez, J., Barry-Ryan, C., and Bourke, P. (2009). Antimicrobial activity of plant essential oils using food model media: efficacy, synergistic potential and interactions with food components. Food Microbiol. 26, 142-150. doi: 10.1016/j.fm.2008.10.008

Hemaiswarya, S., Kruthiventi, A. K., and Doble, M. (2008). Synergism between natural products and antibiotics against infectious diseases. Phytomedicine 15, 639-652. doi: 10.1016/j.phymed.2008.06.008

Hughes, N. E., Marangoni, A. G., Wright, A. J., Rogers, M. A., and Rush, J. W. (2009). Potential food applications of edible oil organogels. Trends Food Sci. Technol. 20, 470-480. doi: 10.1016/j.tifs.2009.06.002

Lv, F., Liang, H., Yuan, Q., and Li, C. (2011). In vitro antimicrobial effects and mechanism of action of selected plant essential oil combinations against four food-related microorganisms. Food Res. Int. 44, 3057-3064. doi: 10.1016/j.foodres.2011.07.030
Ma, Q., Davidson, P. M., and Zhong, Q. (2013). Antimicrobial properties of lauric arginate alone or in combination with essential oils in tryptic soy broth and $2 \%$ reduced fat milk. Int. J. Food Microbiol. 166, 77-84. doi: 10.1016/j.ijfoodmicro.2013.06.017

Moosavy, M. H., Basti, A. A., Misaghi, A., Salehi, T. Z., Abbasifar, R., Mousavi, H. A. E., et al. (2008). Effect of Zataria multiflora Boiss. essential oil and nisin on Salmonella typhimurium and Staphylococcus aureus in a food model system and on the bacterial cell membranes. Food Res. Int. 41, 1050-1057. doi: 10.1016/j.foodres.2008.07.018

Oliveira, J., Cunha, A., Castilho, F., Romalde, J. L., and Pereira, M. J. (2011). Microbial contamination and purification of bivalve shellfish: crucial aspects in monitoring and future perspectives-A mini-review. Food Control 22, 805-816. doi: 10.1016/j.foodcont.2010.11.032

Rhayour, K., Bouchikhi, T., Tantaoui-Elaraki, A., Sendide, K., and Remmal, A. (2003). The mechanism of bactericidal action of oregano and clove essential oils and of their phenolic major components on Escherichia coli and Bacillus subtilis. J. Essent. Oil Res. 15, 286-292. doi: 10.1080/10412905.2003.9712144

Sikkema, J., De Bont, J. A., and Poolman, B. (1995). Mechanisms of membrane toxicity of hydrocarbons. Microbiol. Rev. 59, 201-222.

Sun, J. (2007). D-Limonene: safety and clinical applications. Altern. Med. Rev. 12, 259-264.

Tajkarimi, M. M., Ibrahim, S. A., and Cliver, D. O. (2010). Antimicrobial herb and spice compounds in food. Food Control 21, 1199-1218. doi: 10.1016/j.foodcont.2010.02.003

Weerakkody, N. S., Caffin, N., Turner, M. S., and Dykes, G. A. (2010). In vitro antimicrobial activity of less-utilized spice and herb extracts against selected food-borne bacteria. Food Control 21, 1408-1414. doi: 10.1016/j.foodcont.2010.04.014

Xiao, D., Gömmel, C., Davidson, P. M., and Zhong, Q. (2011). Intrinsic Tween 20 improves release and antilisterial properties of co-encapsulated nisin and thymol. J. Agric. Food Chem. 59, 9572-9580. doi: 10.1021/jf201864v

Zahi, M. R., Wan, P., Liang, H., and Yuan, Q. (2014). Formation and stability of D-limonene organogel-based nanoemulsion prepared by a high-pressure homogenizer. J. Agric. Food Chem. 62, 12563-12569. doi: 10.1021/jf5032108

Zhang, Z., Vriesekoop, F., Yuan, Q., and Liang, H. (2014). Effects of nisin on the antimicrobial activity of D-limonene and its nanoemulsion. Food Chem. 150, 307-312. doi: 10.1016/j.foodchem.2013.10.160

Conflict of Interest Statement: The authors declare that the research was conducted in the absence of any commercial or financial relationships that could be construed as a potential conflict of interest.

Copyright (c) 2015 Bei, Zhou, Xing, Zahi, Li, Yuan and Liang. This is an open-access article distributed under the terms of the Creative Commons Attribution License (CC BY). The use, distribution or reproduction in other forums is permitted, provided the original author(s) or licensor are credited and that the original publication in this journal is cited, in accordance with accepted academic practice. No use, distribution or reproduction is permitted which does not comply with these terms. 